\title{
SOBRE EL ESTUDIO BIOLÓGICO DEL LENGUAJE 50 AÑOS DESPUÉS. UNA CONVERSACIÓN CON NOAM CHOMSKY ${ }^{1}$
}

\author{
Patrick C. Trettenbrein ${ }^{2}$ \\ Max Planck Institute for Human Cognitive and Brain Sciences (Alemania) \\ trettenbrein@cbs.mpg.de
}

Recibida: 26/01/2021 - Aprobada: 25/02/2021 - Publicada: 30/07/2021

DOI: doi.org/10.17533/udea.lyl.n80a21

Entrevista traducida del inglés por Miguel Ángel Mahecha Bermúdez y Rubén Arboleda Toro.

$\mathrm{N}$ o es una exageración considerar a Eric Lenneberg como el fundador del campo de la biología del lenguaje y a sus Fundamentos biológicos del lenguaje [Biological Foundations of Language] como uno de los documentos fundadores de dicho campo. Del mismo modo, la lingüística moderna en la tradición de la gramática generativa fue fundada por Noam Chomsky en la década de 1950 y/con su libro Estructuras sintácticas [Syntactic Structures] como uno de los documentos fundadores de este campo.En sus comienzos, el trabajo de Chomsky y Lenneberg, así como sus respectivos libros seminales, no parecen estar claramente relacionados; después de todo, en Fundamentos biológicos del lenguaje se examinó la literatura biológica, mientras que en Estructuras sintácticas se proporcionó un análisis formal de la sintaxis de las lenguas naturales. Sin embargo, nada podría estar más lejos de la verdad: Lenneberg y Chomsky cofundaron lo que hoy se conoce como biolingüística en la época en que eran estudiantes de posgrado en Harvard. Incluso un rápido vistazo a los Fundamentos biológicos del lenguaje revela lo siguiente: Chomsky contribuyó con un apéndice sobre "La naturaleza formal del

1. Artículo original titulado «50 Years Later: A Conversation about the Biological Study of Language with Noam Chomsky», publicado en la sección Forum de la revista Biolinguistics, 11, 2017, SI: 487-499, http://www.biolinguistics.eu.

2. El profesor Trettenbrein es un científico cognitivo en formación, que trabaja en el laboratorio de Angela D. Friederici en el Instituto Max Planck de ciencias cognitivas y cerebrales humanas en Leipzig, Alemania. Su área de interés investigativa es la neurobiología del lenguaje, centrándose en la modalidad (in)dependencia de los cálculos lingüísticos en el cerebro. En otras palabras, investiga el lenguaje humano como un modo de cognición específico de una especie. Nota del entrevistador: En este documento, todas las preguntas formuladas por el entrevistador (Patrick C. Trettenbrein) se imprimieron en cursiva, mientras que las respuestas del profesor Chomsky se presentan en letra imprenta. Es preciso tener en cuenta que esta entrevista también incluye algunas preguntas planteadas originalmente por colegas y amigos, que el entrevistador ha intentado reproducir aquí con la mayor precisión posible. Se han agregado las referencias a los libros y artículos mencionados para comodidad de los lectores. 
Sobre el estudio biológico del lenguaje 50 años después. Una conversación con Noam Chomsky lenguaje" . Una mirada más atenta revela que el propio Lenneberg se basó en gran medida en el análisis formal (del lenguaje) tal como lo proporcionó Chomsky para avanzar su argumento (en este contexto, véanse los estudios de Piattelli-Palmarini y Becker ${ }^{4}$, publicados en este número especial). Así las cosas, lo más obvio era hablar con Noam Chomsky, siendo este cofundador del campo, contemporáneo y amigo de Eric Lenneberg. Afortunadamente, el profesor Chomsky se tomó el tiempo para responder algunas preguntas sobre los primeros días del campo de la biología del lenguaje, su trabajo y relación con Lenneberg, y otras preguntas y cuestiones científicas que (aún) nos siguen cautivando después de 50 años.

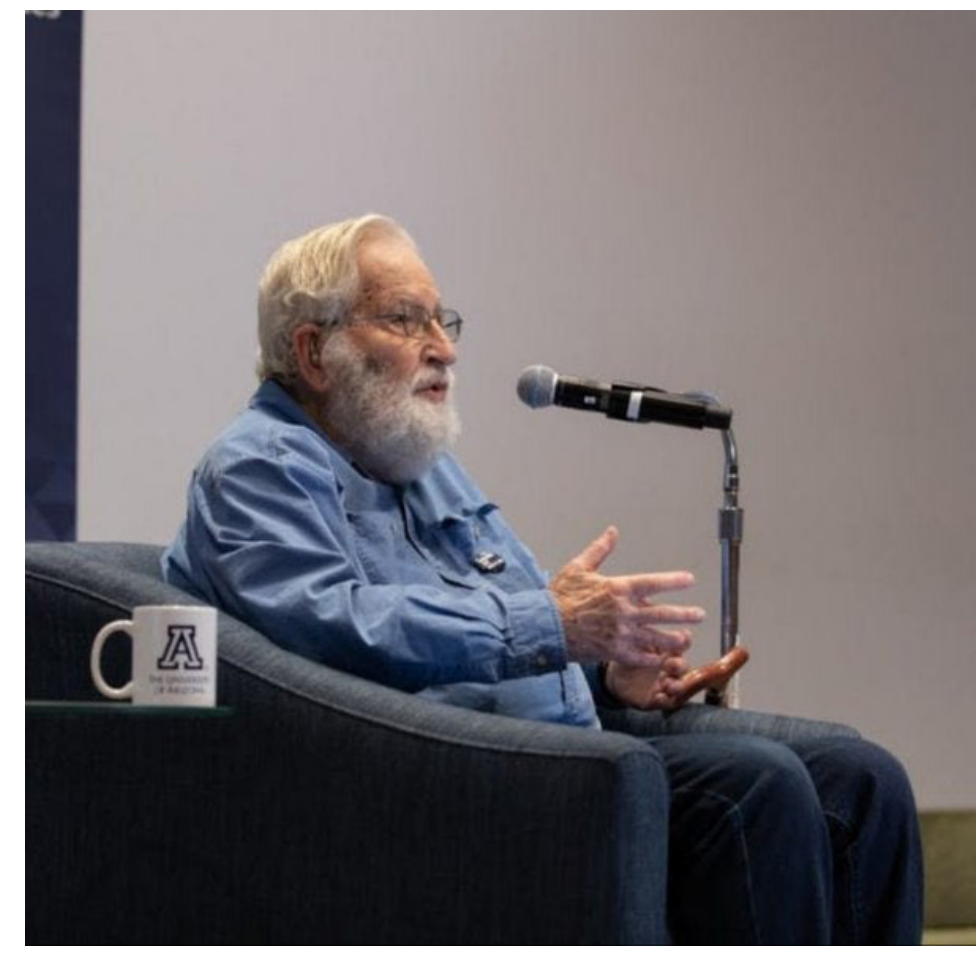

Imagen 1. Noam Chomsky. Fuente: https://www.reddit.com/r/chomsky/comments/ddmv7v/hes_grown_a_beard/

3. Nota de los traductores: Chomsky $(1980,7-8)$ hace una descripción del anexo publicado en 1967: "El capítulo 5 [esto es La naturaleza formal del lenguaje, A\&M] es un estudio considerablemente más técnico, que investiga con algún detalle el material presupuesto o simplemente desarrollado sin rigor en El lenguaje y el entendimiento. En este caso, el público de mi conferencia consistía fundamentalmente en psicólogos y psicolingüistas. Este capítulo, que apareció originariamente como apéndice a Biological Foundations of Language, de Eric Lenneberg, es un intento de dar una presentación sistemática y concisa de la teoría de la gramática generativotransformacional a la vez que una exploración de su importancia potencial para la psicología humana." El libro de Lenneberg tiene dos apéndices, el de Chomsky, ya mencionado, y el de Otto Marx titulado La historia de la base biológica del lenguaje.

4. Nota de los traductores. Massimo Piattelli-Palmarini, From Zero to Fifty: Considerations on Eric Lenneberg's Biological Foundations of Language and Updates, pp. 423-444, sección Forum. Este capítulo fue traducido al español por el profesor Carlos Javier Martínez y se publicó en Mahecha \& Arboleda (2020). La contribución de Misha K. Becker se titula Innate Mechanisms for Acquiring Syntactic Displacement, pp. 381-394, sección Briefs. 
En primer lugar, permitame agradecerle por tomarse el tiempo para responder a mis preguntas. Como usted sabe, la razón de esta entrevista es el cincuentenario de la publicación de la monumental obra de Eric Lenneberg Fundamentos biológicos del lenguaje (1967), considerado ahora sin ninguna duda como uno de los documentos fundacionales del campo, junto con sus Estructuras sintácticas (Chomsky 1957/20025). Me gustaría comenzar preguntándole acerca de su relación con Eric Lenneberg en los primeros días de la biolingüística. Supongo que ustedes dos se conocieron por primera vez durante su estancia en Harvard.

Llegué a Harvard en 1951. Eric lo hizo casi al mismo tiempo. Nos conocimos muy pronto y rápidamente nos hicimos amigos cercanos, así como nuestras familias.

¿Qué tan cerca estuvo trabajando con Eric Lenneberg en esos primeros días (y también luego)? Al releer Fundamentos biológicos del lenguaje a fines del año pasado, me sorprendió lo modernos que eran muchos de sus puntos de vista a pesar de los limitados datos con los que pudo contar. Del mismo modo, me sorprendió la precisión con la que muchos de los argumentos que presenta se alinean con lo que yo asumo que son sus puntos de vista. Consideremos, por ejemplo, algunos de los puntos discutidos por Massimo Piattelli-Palmarini (ver su contribución): la versión de Lenneberg sobre el desarrollo del lenguaje, sus consideraciones sobre una posible genética del lenguaje, o sobre una base de un nivel más conceptual, la distinción entre competencia y actuación; Incluso sus ideas sobre la evolución del lenguaje. ¿Qué tan grande consideraría la influencia de Lenneberg en su pensamiento sobre estos problemas (e incluso más allá)?

Pasamos mucho tiempo juntos a principios de la década de 1950, junto con nuestro amigo mutuo Morris Halle, discutiendo algunos intereses comunes, el trabajo que estábamos haciendo, los problemas que estábamos planteando, los artículos y libros que estábamos estudiando, y el estado de las áreas de nuestro interés. Y mucho más. Lo que nos unió a este respecto fue un escepticismo compartido sobre gran parte de lo que era la ortodoxia virtual en ese momento en Cambridge: el conductismo radical y la ciencia del comportamiento en general en las formas que estaba tomando, las doctrinas reinantes de la lingüística estructural, la euforia sobre las perspectivas de nuevas tecnologías, y una actitud prevaleciente, que nos parecía errónea, que estos supuestos avances pronto revolucionarían el estudio del pensamiento y el comportamiento humanos, expulsando el misticismo mentalista tradicional.

Eric había comenzado su investigación de la biología del lenguaje en esos años, pero las ideas que usted describe aquí, y así hay muchas, provienen principalmente del trabajo de sus años posteriores. A mediados de la década de 1950, Eric y yo abandonamos Harvard. Él con destino a la escuela de medicina, y yo al Instituto de

5. Nota de los traductores: En el 2002, la editorial Mouton de Gruyter (Berlín, New York), publica la segunda edición de este clásico con una Introducción (actualización) de David W. Lightfoot. 
Sobre el estudio biológico del lenguaje 50 años después. Una conversación con Noam Chomsky

Tecnología de Massachusetts (MIT). Nos mantuvimos en contacto, pero no con la misma intensidad de comienzos de los años 50, cuando éramos estudiantes graduados de Harvard y nos reuníamos con regularidad.

A pesar de la enorme superposición entre sus puntos de vista, hasta donde he podido darme cuenta, ustedes nunca terminaron publicando un artículo en conjunto, aunque, como sabemos, usted escribió un apéndice para Fundamentos biológicos del lenguaje. En muchos aspectos, su trabajo y el de Eric Lenneberg parecen complementarios en la medida en que usted se enfocó en varios temas conceptuales y adoptó un enfoque teórico respectivamente más computacional mientras que él decidió profundizar en biología. ¿Cómo es que ustedes dos nunca terminaron publicando juntos un artículo?

Durante los años en que estuvimos en contacto cercano éramos estudiantes de posgrado. Años después, las posibilidades de publicación se fueron limitando debido al tipo de trabajo en el que cada quien estaba comprometido, el cual, por cierto también, estaba bastante aislado. Y para cuando aparecieron las oportunidades de publicación íbamos por caminos en cierta medida separados, si bien, relacionados.

De manera general, ¿podría hablar un poco de los primeros días? Qué los impulsó a usted y a Lenneberg a plantearse estas cuestiones, es decir, ¿qué los llevó a mirar el lenguaje desde una perspectiva biológica?

Para nosotros tres, Eric, Morris y yo, simplemente parecía obvio que la facultad humana del lenguaje hace parte de la biología humana, al igual que lo visual y otras facultades. Si es así, solo tiene sentido tratar de incorporar el estudio del lenguaje dentro de un marco biológico general; fue la vía que Eric siguió a su manera con notable intensidad y éxito.

Con respecto a Eric Lenneberg como persona, ¿recuerda usted qué provocó su interés específico en el lenguaje o de dónde pudo venir ese interés? Es más, ¿tiene alguna idea de por qué decidió estudiar medicina después de haber obtenido su doctorado en lingüistica y psicología?

Cuando nos conocimos en 1951, cada uno de nosotros tenía un interés especial en el lenguaje y la psicología. Después de que Eric terminó sus estudios en lingüística-psicología, dada su particular preocupación por los fundamentos biológicos, era bastante natural para él entrar a la carrera de Medicina.

Esta puede ser una pregunta un tanto extraña, porque, como sabemos, usted escribió un capítulo sobre la naturaleza del lenguaje que se incluyó como un apéndice en Fundamentos Biológicos de Lenguaje, lo que significa que obviamente respaldó el libro y lo que Lenneberg afirmó sobre el lenguaje y la biología; no obstante, me gustaría saber cuál fue su impresión general cuando el libro apareció por primera vez ¿cuándo fue la primera 
Sobre el estudio biológico del lenguaje 50 años después. Una conversación con Noam Chomsky vez que leyó el manuscrito?

Pensé que sería un clásico de inmediato, básicamente que fundaba una nueva disciplina. Es sorprendente, inquietante inclusive, que no se conozca mejor. Solo por mencionar un ejemplo, ha habido un enorme interés en la evolución del lenguaje desde los años 90 — muchas publicaciones, conferencias, sociedades_-, pero es bastante raro encontrar una referencia a la discusión que Eric planteaba sobre el tema en su libro clásico, a pesar de que fue pionero en su momento y sigue siendo hoy una de las aportaciones más sofisticadas y avanzadas sobre el tema.

De acuerdo. Ahora, mirando en retrospectiva 50 años atrás, ¿cuál considera usted que es el mayor logro del libro? ¿Por qué cree que sigue siendo relevante y por qué los investigadores y los estudiantes actuales deberían tomarse la molestia de leerlo?

El libro desarrolló una fundamentación biológica sólida, profundamente informada, completa y cuidadosamente ejecutada, para el estudio de la facultad del lenguaje humano, su uso y su evolución. No sólo reúne lo que se sabía sobre este tema sino que lo acrecienta sustancialmente e incluye provocativas especulaciones que estaban lejos de ser convencionales en su tiempo pero que desde entonces en muchos casos se ha demostrado que han estado en el camino correcto. Y si bien se ha aprendido mucho desde entonces, este estudio sigue siendo una fuente indispensable de investigación sobre la biología del lenguaje en todos sus aspectos.

Sí, es impresionante cómo la visión moderna de Lenneberg sobre el desarrollo del lenguaje y la teoría biológica del lenguaje que esboza al final del libro en un capítulo de síntesis ${ }^{6}$ estaban ya a pesar de que gran parte del trabajo empírico importante sobre adquisición a la que podemos aproximarnos hoy aún no se había realizado. Si bien algunas modificaciones y actualizaciones pueden ser necesarias aquí y allá, su teoría es todavía muy precisa. En trabajos anteriores (Lenneberg 1964) indicó que su objetivo a largo plazo era hacer exactamente esto: alcanzar una teoría biológica del lenguaje.

Aun así, es interesante notar que Fundamentos biológicos del lenguaje de muchas maneras llegó un poco tarde a la fiesta: La reseña que usted le hizo a Verbal Behavior (Chomsky, 1959) ya había tenido un gran efecto en la lingüistica y la psicología y algunos reseñistas del libro de Lenneberg señalaron en su momento que, de alguna manera, parece que no solo trató de esbozar una teoría biológica del lenguaje, sino que también quería,

[...] proporcionar una plausibilidad biológica tangible para conclusiones con las que un grupo de Empiricistas inconformes [...] [se había] comprometido sólo sobre la base de un argumento formal. (Bem \& Bem 1968: 498499).

6. Nota de los traductores: Se trata del capítulo 9: Hacia una teoría biológica del desarrollo del lenguaje. (Resumen general). 
Sobre el estudio biológico del lenguaje 50 años después. Una conversación con Noam Chomsky ¿Está de acuerdo?

Cuando llegamos a Harvard, las conferencias William James de Skinner de 1948, que constituían la esencia de su libro Verbal Behavior, fueron ampliamente consideradas como el enfoque definitivo de la psicología del lenguaje, en parte debido a la adopción del marco básico por el influyente filósofo de Harvard, W.V.O. Quine. Nuestro triunvirato estuvo prácticamente solo al considerar que dicha posición estaba seriamente equivocada.

Mi reseña, a la que usted hace referencia, se envió para su publicación en 1957, cuando Eric ya estaba inscrito en la escuela de medicina, pero habíamos discutido estos asuntos ampliamente años atrás. La revisión se basó en nuestra lectura y discusión de la literatura etológica que estaba alejada de la ortodoxia reinante. La rigidez de dicha ortodoxia se ilustra por el hecho de que incluso el importante artículo de Karl Lashley de 1951 sobre el orden serial en el comportamiento, que socavó bastante el conductismo skinneriano, era aparentemente desconocido en la comunidad de ciencias del comportamiento de Cambridge. No pude encontrar una referencia en la literatura relevante cuando lo mencioné en la reseña (y supe de él por un historiador del arte, Meyer Schapiro). Pero estos eran el tipo de temas que estábamos discutiendo. La exploración de Eric de los fundamentos biológicos ya estaba en marcha en ese momento, aunque completamente desarrollado y publicado sólo unos años después.

Entonces, ustedes comenzaron leyendo en Harvard la literatura etológica europea en el original alemán, porque Lenneberg creció en Alemania. Como se sabe, usted señaló que el lenguaje se estudia mejor dentro de un marco etológico y hay algunos puntos obvios sobre el lenguaje que se pueden considerar y que todavía no tienen reconocimiento universal. Por ejemplo, la idea clara y directa de que su lengua es una propiedad suya y, ante todo, que su cerebro es considerado controversial todavía, — quizás de manera implícita-, cuando hay cientificos cognitivos que dicen que las lenguas evolucionan para adaptarse a los cerebros humanos (por ejemplo, Christiansen \& Müller, 2015), sea lo que sea que se quiera significar. ¿Cuál es su opinión sobre el estado actual del programa de investigación biolingüistica?

Es cierto: “sea lo que sea que se quiera significar" Y sí, a principios de los años 50, Morris, Eric y yo estuvimos leyendo literatura etológica. Ellos manejaban el alemán y podían leer los originales. Me dediqué principalmente a la literatura en lengua inglesa. Este era territorio extraño en la comunidad intelectual de Cambridge de la que formábamos parte, y en la medida que fue conocido, también fue desestimado. La idea de que podría haber un elemento instintivo en el lenguaje humano, o en las capacidades cognitivas en general, que nos pareció un truismo, fue considerado como un disparate por las figuras más influyentes. Así que fue una preocupación privada. Casi. George Miller fue receptivo a lo que estábamos pensando, junto con varios compañeros de estudios. Pero muy pocos.

En los años transcurridos desde entonces, y en la actualidad, la biolingüística ha estado floreciendo, gracias a una comprensión mucho más profunda de la naturaleza del lenguaje, su adquisición y uso, pero también en parte 
Sobre el estudio biológico del lenguaje 50 años después. Una conversación con Noam Chomsky gracias a la nueva tecnología de las imágenes internas del cuerpo humano (la imagenología) que ha superado en cierta medida las restricciones éticas en la experimentación directa; en cambio, a diferencia del estudio de la visión y otras capacidades, los experimentos con otros animales nos dicen muy poco debido a la especificidad de especie de las propiedades básicas del lenguaje. No hay sistemas homólogos que nos permitan avanzar en la investigación.

Divagando un poco solo por un segundo, me gustaría hacerle una pregunta relacionada con la lingüística como un campo más general. Sus ideas y por lo tanto, por extensión, las ideas de Eric Lenneberg sobre el estudio cientifico de la facultad del lenguaje a veces se presentan todavía como controvertibles incluso hoy, aunque es interesante ver que casi nunca se hace referencia a Lenneberg por parte de los criticos a pesar de haber defendido un punto de vista muy similar. ¿Qué opina de esto? Uno pensaría que sería más atractivo atacar a alguien que ya no está presente para defender sus puntos de vista.

No creo que esa sea la razón. Hay que preguntar de qué críticos se trata. En los últimos 60 años, he discutido con personas y audiencias de diferentes disciplinas el tipo de trabajo que nosotros (y actualmente muchos más) hemos venido haciendo. Es a menudo considerado altamente controversial (si no absurdo) por los filósofos, lingüistas, y una variedad de científicos sociales, pero no por físicos, matemáticos, biólogos, incluyendo figuras distinguidas, entre ellas premios Nobel en biología evolutiva, con uno de los cuales co-orienté seminarios de posgrado en biología del lenguaje en los años setenta ${ }^{7}$.

Entre los críticos, el trabajo de Eric era apenas conocido, si es que se conocía, y si hubiera sido conocido habría sido considerado «ciencia real», no sujeto a este tipo de críticas. En las ciencias duras, las teorías explicativas que se desarrollan en lo que David Marr (1982/2010) llamó niveles computacional/representacional y algorítmico se consideran bastante naturales, y las prácticas similares son familiares en las disciplinas. Estos son asuntos que han sido ampliamente discutidos. Para mencionar un ejemplo, Jerry Fodor ha dedicado gran parte de su distinguida carrera a explicar la validez de tales enfoques y a contrarrestar la crítica de ellos ${ }^{8}$

Continuando un poco más con este tema, parece que hay dos formas diferentes de hacer lingüística, en algún nivel tal vez análogo a la forma en que la biología era hecha cuando todavía se llamaba historia natural en oposición a cómo se lleva a cabo la investigación biológica hoy en día. Norbert Hornstein habla de langüística a diferencia de lingüística9 ${ }^{9}$ Para precisar, ¿cree usted que toda la disciplina está atrapada todavía en la etapa

7. Nota de los traductores: Se trata de Salvador Luria. Nació en 1912 en Turín (Italia) y falleció en Boston (Estados Unidos) en 1991. Véase su obra A Slot Machine, A Broken Test Tube. An Autobiography (Harper \& Row, New York, 1984).

8. Nota del entrevistador: este intercambio tuvo lugar antes del fallecimiento de Jerry Fodor al final de noviembre de 2017.

9. Nota de los traductores: Para entender la diferencia propuesta por Norbert Hornstein entre «langüística» y «lingüística», véase Hornstein's lament en http://facultyoflanguage.blogspot.com/2014/03/hornsteins-lament.html 
Sobre el estudio biológico del lenguaje 50 años después. Una conversación con Noam Chomsky de «historia cultural», catalogando lo que se encuentra en el mundo, de la misma forma que lo hicieron los antecesores de los biólogos cuando la biología era todavía historia natural?

La respuesta se vuelve clara cuando se compara lo que aparecía en las revistas en los años 50 con lo que aparece en las (muchas más) revistas de hoy. Ilustra dramáticamente un cambio de langüística a lingüística en el sentido de Hornstein. Además, La gama tipológica de lenguas investigadas se ha extendido enormemente con la profundidad de las preguntas examinadas, la mayoría de ellas inimaginables hace tan solo algunos años. En este sentido crucial, entonces, «catalogar lo que se encuentra en el mundo» se ha ampliado enormemente tanto en alcance como en profundidad.

Volviendo a mi pregunta anterior sobre la relación de su trabajo con el de Eric Lenneberg, me gustaría detenerme un poco más en la relación de la lingüistica y la biología. Mientras que el enfoque biológico para estudiar el lenguaje tomó forma en los años 50 y 60 del siglo pasado, aparentemente la captación de la etiqueta «biolingüística» tomó tiempo y sólo se popularizó en las últimas décadas, entre otras cosas con el establecimiento de Biolinguistics, la revista en la que se publicará esta entrevista. Recientemente, algunas personas se han quejado de que el nombre de biolingüistica es una especie de cambio de marca de la gramática generativa, mientras que la biolingüística real debe entenderse más exclusivamente como una etiqueta para toda la investigación biológica de la facultad del lenguaje (por ejemplo, Martins \& Boeckx, 2016). ¿Cuál es su opinión sobre esto?

No lo veo como un problema. La investigación biológica de la facultad del lenguaje es, por definición, un enfoque para la investigación de la facultad del lenguaje. La Gramática generativa es el estudio de las propiedades fundamentales de la facultad del lenguaje. ¿Por qué tendría que haber algún problema?

Estoy de acuerdo. Tal como lo entendí, la crítica es sobre lingüistas teóricos que en gran medida continúan con sus trabajos «usuales»y sin embargo los denominan «biolingüistica», a pesar de no buscar activamente en ellos la integración con la biología. Boeckx y Grohmann (2007), en el artículo inaugural de esta revista, hicieron referencia a estos dos sentidos de la biolingüística: el «débil» y el «fuerte»; el primero referido a los lingüistas que todavía buscan descubrir las propiedades de la gramática, y el segundo, al trabajo que requiere la integración de conocimientos lingüísticos con los de otras disciplinas. ¿Está usted de acuerdo en que esta distinción debería hacerse?

Los individuos pueden elegir sus propios intereses y proyectos de investigación. Claramente, sin embargo, el dominio de la biolingüística «fuerte», tal como se define, sólo puede perseguirse en la medida que «los conocimientos lingüísticos», — esto es, las «propiedades de la gramática»— se hayan desarrollado lo suficiente como para ser combinados e integrados. Lo mismo en términos generales, sea cual sea el dominio específico de la 
Sobre el estudio biológico del lenguaje 50 años después. Una conversación con Noam Chomsky indagación: la visión (tal como lo discutió David Marr, véase Marr 1982/2010), la comunicación de los insectos, o cualquier otra. Una vez más, no veo ningún problema.

Al volver a leer muchas de las publicaciones de Eric Lenneberg como preparación para este proyecto, me pareció que él probablemente habría estado de acuerdo con la evaluación bastante crítica de que todavía falta una verdadera integración de la lingüística y la biología y que la gramática generativa ha «secuestrado» la etiqueta biolingüistica. Obviamente, había una brecha clara entre lingüística y biología, tanto en los días de Lenneberg como hoy en día. ¿Fue él optimista sobre una eventual integración de la lingüística y la biología? Y ¿es usted mismo optimista sobre eso y cree que ahora estamos más cerca de una «biolingüística real» que hace 50 años?

Como se señaló, no entiendo el asunto del «secuestro». Seguramente se ha aprendido mucho sobre la biología del lenguaje — biolingüística — en los últimos 50 años. Yo no sé lo que es una «biolingüística real» más allá de lo que sabría que es una biología real de la visión. Hay una creciente comprensión de estos temas. ¿Qué más podemos esperar?

Admito que «secuestro» puede ser un término un poco exagerado. Aun así, en realidad tenía una cita de Lenneberg en mente. Él escribió que,

nada se gana con etiquetar la inclinación a ver el lenguaje como realidad biológica a menos que podamos usar esta información para nuevas direcciones de investigación, a menos que se puedan descubrir correlatos más especificos (Lenneberg 1964:76)

Supongo que usted diría que la gramática generativa ofrece «nuevos caminos de investigación» ¿y es por eso que el problema ni siquiera surge?

Cuando una lengua se entiende como un objeto biológico, como sucede en la gramática generativa (una lengua-I en terminología contemporánea), entonces surgen ciertas preguntas:

- ¿Cómo se adquiere el lenguaje?

- ¿Cuáles son sus bases neurales?

- ¿Cómo evoluciona?

- ¿Cómo se usa?

Tales preguntas no pueden formularse de forma clara si el lenguaje se considera como algún tipo de propiedad de la comunidad, por ejemplo, un «tipo de contrato» en una comunidad (Saussure) o «la totalidad de los enunciados 
Sobre el estudio biológico del lenguaje 50 años después. Una conversación con Noam Chomsky

realizados en una comunidad de habla» (Bloomfield). En consecuencia, aunque no se dejen de lado por completo, tales preguntas sólo pueden ser abordadas por vías circunscritas a dichas concepciones.

Además, como ya se señaló, es obvio que tales preguntas puedan ser consideradas seriamente solo en la medida en que las propiedades de estos objetos biológicos sean entendidas. No es controvertido que estas «nuevas vías de investigación» se hayan desarrollado en formas altamente productivas dentro del «marco biolingüístico» general que la gramática generativa adoptó desde sus orígenes.

Frente a este contexto, considere la siguiente cita de un informe de Eric Lenneberg escrito en 1972, rescatado del olvido por Michael Arbib (cf. Arbib, en este número de la revista ${ }^{10}$ ), unos años después de la publicación de Fundamentos biológicos del lenguaje, que incluye su apéndice:

En la actualidad, solo hay un tipo de teoría cientifica de la estructura del lenguaje disponible, la gramática generativa, y esta nunca tuvo la intención de servir como modelo para los mecanismos biológicos. La intención era crear un formalismo que describiera adecuadamente la red de relaciones que caracteriza una lengua natural tal como el inglés. Durante un tiempo parecía que podría convertirse en un algoritmo del análisis de lenguaje, pero esto es todavía un sueño no cumplido (excepto para un discurso altamente restringido). La gramática generativa nos ayuda aqui y allá a definir el significado de la complejidad en la estructura de las oraciones, pero debido a la forma en que se configura, también clasificará como complejas (es decir, productos de un larga y compleja historia de transformaciones) algunas expresiones que son intuitivamente las más simples de todas, por ejemplo, «iAgua!», que tendría que considerarse como un derivado de «¡Dame agua!» que, a su vez, se deriva de una forma más básica, «*Usted deme agua». Lo que se necesita con mayor urgencia es una teoría de la producción y comprensión de oraciones que tenga la precisión formal del enfoque de Chomsky, pero es con la intención explícita de explicar los fundamentos psicobiológicos de las capacidades del lenguaje (Lenneberg 1972, pp.635-636).

¿Cree usted que los desarrollos de la gramática generativa en las últimas décadas están más cerca de la teoría de la producción y comprensión de oraciones que Lenneberg indicó? En muchos sentidos, el trabajo dentro del Programa Minimalista (PM) parece estar alejado de una teoría de la producción y procesamiento del lenguaje y enfocado, en vez de eso, en la naturaleza formal de la maquinaria involucrada.

No entiendo completamente la cita. Una gramática generativa $\mathrm{G}$ de la lengua L busca determinar la estructura de la clase infinita de expresiones de L y su interpretación en la interfaz conceptual-intencional (semánticopragmática) y sensoriomotor — el papel de la interfaz conceptual-intencional es primario, como lo sugiere un trabajo reciente - . Es una teoría de la Competencia. Las teorías de la actuación para L, accederán por supuesto a la competencia almacenada caracterizada por G; y las teorías generales de la actuación accederán en consecuencia

10. Nota de los traductores: Language and Brain: Developmental Aspects: Eric Lenneberg at the Neurosciences Research Program in 1972. Michael A. Arbib, pp. 463-480, sección Forum. 
Sobre el estudio biológico del lenguaje 50 años después. Una conversación con Noam Chomsky

a las propiedades generales de las gramáticas de la competencia. Todo cae dentro de la biología, y todo tiene «fundamentos psicobiológicos».

Ha habido un gran progreso en el estudio del procesamiento del lenguaje, incluyendo un trabajo extenso y productivo sobre analizadores minimalistas, gran parte de él aparecerá en un próximo libro sobre analizadores minimalistas editado por Robert Berwick (Berwick \& Stabler, en prensa ${ }^{11}$ ). El estudio de la producción del lenguaje también ha progresado igualmente, con una gran brecha que se mantiene para toda acción voluntaria. Como se describe en sentido figurado por dos destacados investigadores de la acción voluntaria, Emilio Bizzi y Robert Ajemian (Bizzi \& Ajemian, 2015), estamos aprendiendo mucho sobre el títere y los hilos, pero el papel del titiritero sigue siendo un misterio. En el caso del lenguaje, fue esencialmente este misterio el que inspiró el asombro y la maravilla de algunos de los grandes fundadores de la ciencia moderna, incluidos Galileo, Descartes y los lógicos-lingüistas de Port Royal.

Por supuesto, estoy de acuerdo en que todo se enmarca en la biología, las teorías de la actuación y la competencia. En lo que entiendo de la cita, Lenneberg estaba señalando, en parte, la discrepancia entre lingüística y biología que ya discutimos brevemente. Parece que el estudio de la competencia ha aislado y aún aísla a la lingüística de la psicología y la neurociencia; ¿tal vez incluso evitando una integración más estrecha de estos campos respectivos, como en partes de la psicolingüística?

Chesi y Moro (2015) han argumentado recientemente que la competencia y la actuación son en realidad interdependientes. Yo diría que hay esencialmente tres escenarios posibles en que la relación de gramática $(G)$ y un analizador sintáctico como sistema de actuación (P) podría funcionar fuera: (i) G podría ser independiente de P, (ii) P podría acceder a $G$ en línea durante el procesamiento, o (iii) podría resultar que G solo se implementa en el software en la medida en que la totalidad de los mecanismos de P dan lugar a un sistema que se comporta de una manera que es capturada por la descripción de G. ¿Qué piensa sobre esto? ¿Y cómo describiría la relación de la lingüística con la psicología y la neurociencia?

No entiendo nada de esto. El estudio de la competencia no puede aislarse de la psicología porque es parte de la psicología, a menos que (perversamente) definamos «psicología» excluyendo el conocimiento almacenado internamente del lenguaje, la aritmética, etc. La psicolingüística, durante los últimos 50 años, se ha integrado estrechamente con el estudio de la competencia lingüística. ¿Cómo podría ser de otra manera? Lo mismo con la neurolingüística. La competencia lingüística está representada en el cerebro — no en el pie, o en el espacio exterior- y lo mismo ocurre con las actuaciones que acceden a este conocimiento almacenado del lenguaje.

Hablando personalmente, siempre he considerado la lingüística, al menos los aspectos que me interesan,

11. Nota de los traductores: Como lo mencionó el entrevistador en la nota 1, se cita en las referencias los textos mencionados en la entrevista. Para el caso de Berwick \& Stabler, el título que indica (Parsing with Minimalist Grammars) no corresponde al libro que fue publicado y cuya referencia precisamos: Robert C. Berwick and Edward P. Stabler (Eds.) 2019. Minimalist Parsing. NewYork, Oxford University Press. 
Sobre el estudio biológico del lenguaje 50 años después. Una conversación con Noam Chomsky como parte de la psicología, y en última instancia, de la biología. La relación de la lingüística con la psicología es similar a la relación de la teoría de la visión con la psicología: parte a todo. Y en la medida en que nos preocupa lo que está sucediendo en el cerebro, se integra con la neurociencia. En síntesis, no entiendo cómo surgen aún algunas de estas preguntas, excepto bajo la delimitación de campos que parecen bastante arbitrarios y nunca han tenido sentido para mí.

Por supuesto, estoy de acuerdo en que la competencia lingüistica no está representada en el pie. A lo que yo trataba de llegar es a que, por ejemplo, podría darse el caso de que (a )este conocimiento almacenado internamente solo se podía acceder durante el procesamiento, en el sentido de que está integrado en la forma en que el sistema de actuación funciona. ¿Tiene algún sentido o estoy completamente fuera de lugar?

Se accede a la lengua-I (competencia lingüística) en cada uso del lenguaje: en el procesamiento del input lingüístico, pero también en la producción —incluida la construcción interna de pensamiento-. Si está «integrado en la forma en que funciona el sistema de actuación», entonces debe duplicarse en cada sistema de actuación, lo que no parece una propuesta razonable. No veo ninguna forma de reformular esta idea que no sea la de suponer que la lengua-I es un sistema central al que acceden los sistemas de actuación, de la misma forma que se accede al conocimiento de la aritmética en el cálculo.

Bueno. Recientemente usted fue coautor de un artículo sobre la base neuronal del procesamiento del lenguaje con Angela Friederici (Friederici et al., 2017), por lo que debe ser algo optimista de que se puede lograr un progreso real en esta área. ¿Cuál es su consejo para los investigadores que intentan conectar teoría lingüistica y psicología o neurociencia?

No es exacto. Contribuí con algunos comentarios introductorios a su libro muy importante (Friederici 2017a). Por lo demás, no creo que la formulación de las preguntas sea provechosa, no lo es más que la pregunta de cómo aconsejar a los investigadores que intentan conciliar la teoría de la percepción visual y la psicología además de la neurociencia. No me parece la forma correcta de formular los problemas. Hay diferentes enfoques para el estudio del lenguaje (visión, etc.) y problemas intrigantes en donde se cruzan. El consejo es dedicarse a ellos.

En una nota relacionada, uno de los mayores éxitos del PM ha sido reducir la complejidad de la maquinaria cognitiva postulada, por ejemplo, al depender solo de una sola operación que creemos que está en el núcleo de la facultad del lenguaje, es decir, la Fusión (Merge). Ahora, usted mismo especuló que no es claro del todo si Merge es especifico del lenguaje, al menos en términos filogenéticos. Con respecto a cómo se puede implementar una operación como Merge en wetware (cerebro) ${ }^{12}$ todavía estamos en una situación en la que, en el mejor de los

12. Nota de los traductores. Wetware puede entenderse como el cerebro humano o las células del cerebro humano o los procesos de pensamiento considerados análogos o en contraste con los sistemas informáticos. 
Sobre el estudio biológico del lenguaje 50 años después. Una conversación con Noam Chomsky casos, podemos hacer conjeturas serias (véase, por ejemplo, la contribución de Friederici en este número ${ }^{13}$ ), ya que carecemos de una teoría de vinculación entre lo computacional-algorítmico y el nivel de implementación. Me parece bastante probable un escenario en el que los circuitos capaces de computación recursiva puedan haber evolucionado para otro propósito (por ejemplo, planificación motora o música) y posteriormente hayan sido exaptados ${ }^{14}$ por el lenguaje. ¿Cuál es su opinión actual sobre estos asuntos?

A menudo se afirma que el cómputo recursivo está involucrado en la planificación motora, pero eso me parece un malentendido, tanto del cálculo recursivo como de la planificación motora. La computación recursiva es válida para sistemas de infinito digital. La planificación motora no es un sistema de infinito digital —aunque se puede imponer una red digital arbitraria en sistemas continuos, dejando los problemas donde estaban- Y como uno mira más allá de esta distinción inicial —y crucial—, las divergencias proliferan.

En cuanto a la música y el lenguaje, me parece mucho más razonable suponer que la música en la medida muy limitada en que ella involucra (un) cómputo recursivo era extraída del lenguaje y no lo contrario. O, tal vez, como lo sugirieron Jeffrey Watumull y Marc Hauser en un trabajo reciente (Hauser \& Watumull, 2016), que el cómputo recursivo surgió y se aplicó en sistemas cognitivos de infinito digital, lenguaje y aritmética, tal vez música.

¿Qué pasa con las etiquetas, la Etiqueta? Recientemente usted sostuvo que la Fusión más simple en contraste con la definición original de Fusión no puede producir objetos etiquetados por necesidad conceptual, y continuó especulando que las etiquetas pueden no existir como objetos sintácticos. ¿Podría dar más detalles sobre esto? ¿Y qué pasa con la endocentricidad, la nuclearización como una propiedad clave del lenguaje humano, después de todo, está en el corazón de la teoría de la X-barra?

Todo esto nos lleva en direcciones diferentes y más técnicas, no apropiadas para este contexto, me parece.

Bueno, algunos colegas me han pedido que le formule algunas preguntas. Uno de ellos pregunta por qué usted está tan convencido de que los sistemas sensoriomotores solo juegan un papel auxiliar en la lengua, esto especialmente cuando se toma una perspectiva de procesamiento. En el nivel computacional, esta visión es por

13. Nota de los traductores. Friederici, Angela D. Neurobiology of Syntax as the Core of Human Language, pp. 325-338, sección Briefs. (Es autora de este artículo y de un libro, que es al que hace referencia el profesor Chomsky, ambos mencionados en la bibliografía de este artículo).

14. Nota del traductor. Palabra sin equivalencia en español. De acuerdo con el diccionario electrónico Merriam-Webster (2021), la palabra exaptation tiene dos significados: 1): «a trait, feature, or structure of an organism or taxonomic group that takes on a function when none previously existed or that differs from its original function which had been derived by evolution.» 2) «the condition or circumstance of possessing one or more such traits, features, or structures.» Según la información que aparece en la misma entrada, este vocablo fue acuñado en los años 80 por los científicos Stephen Jay Gould and Elizabeth Vrba, sugerido como alternativa a preadaptation. En la actualidad, tanto exaptation como preadaptation siguen siendo ampliamente aceptadas en su uso. 
Sobre el estudio biológico del lenguaje 50 años después. Una conversación con Noam Chomsky supuesto muy plausible; pero cuando miramos su implementación, ella (la colega) ya no está tan segura. Piense en la cognición como función corporal. Además, ella piensa que en el contexto del PM estos sistemas auxiliares hacen mucho más trabajo en las interfaces de lo que se suponía anteriormente

He explicado en otros lugares las razones tanto conceptuales como empíricas — para la revisión de algunas de ellas, véase What Kind of Creatures are We? de Chomsky (2016)_. Para mí ellas son sólidas y convincentes. En el procesamiento, los sistemas sensoriomotores juegan, por definición, un papel central. No entiendo el resto. ¿«Más trabajo» en qué? «Que previamente asumido» cuándo? ¿Implementación de qué? No veo qué más hay en la mira.

Bueno. Enseguida, otro colega quería que le preguntara su opinión sobre el origen de los elementos del léxico que, por cierto, tienen mucho trabajo en la teoría sintáctica actual. Lenneberg ya presentó un enfoque de la semántica léxica que no era referencial sino completamente intensional, la importancia de la cual ha usted enfatizado en repetidas ocasiones. En Why Only Us? Language and Evolution (Berwick \& Chomsky, 2016), los elementos léxicos se dejaron de lado casi por completo. Curiosamente, Lenneberg parece haber pensado que la forma en que los humanos clasifican no es cualitativamente diferente de la de otros animales. Además, consideró «palabras» no como etiquetas para conceptos almacenados en la memoria, sino como etiquetas para procesos de categorización. ¿Cuál es su perspectiva actual sobre estas cuestiones? ¿El origen evolutivo de las «palabras» seguirá siendo misterioso o eventualmente pueden ser susceptibles de estudio?

Creo que hay pruebas muy sólidas, que ya he discutido en otra parte, de que los conceptos humanos/semántica léxica son radicalmente distintos de cualquier cosa conocida en otras partes del mundo animal. Su origen evolutivo es un misterio, por lo que fueron «dejados a un lado» en nuestra discusión sobre la evolución de la facultad del lenguaje. Por el momento, hasta donde sé, no hay ideas útiles sobre cómo investigar este misterio.

Por último, si no me equivoco, usted comenzó en el MIT como parte de un proyecto de traducción automática en el que realmente nunca terminó trabajando porque pensaba que no tenía sentido, ¿es correcto? Un colega mío está interesado en la traducción y, aunque no está relacionado en absoluto con el tema de este número especial, quería que también le preguntara ¿cómo cree que uno podría abordar el estudio de la traducción dentro de un marco como el de la gramática generativa? ¿Hay algo así como una «facultad de traducción»? Personalmente, podría agregar que esta pregunta probablemente tiene que ver con cómo concebimos y entendemos el mapeo a la interfaz semántica pragmática y el grado de variación que este mapeo permite en comparación con el mapeo a los sistemas sensoriomotores.

Por casualidad fui nombrado en un proyecto de investigación de traducción automática, pero nunca trabajé 
Sobre el estudio biológico del lenguaje 50 años después. Una conversación con Noam Chomsky en el tema. Desde el principio tuve la sensación de que, para fines prácticos, simplemente es más conveniente hacer que algo que funcione, sin importarnos si aprendemos algo de ello. Si bien algún día la comprensión del lenguaje podría contribuir materialmente a este proyecto, ese día está aún muy lejano. Y la investigación sobre la traducción automática no me pareció la forma de avanzar en el proyecto de comprender la naturaleza del lenguaje. Estas expectativas se han confirmado, hasta donde sé. No conozco ninguna razón para suponer que existe una «facultad de traducción». La cuestión de si la variación en la interfaz semántico-pragmática/conceptual intencional es interesante, en el límite de la investigación —mi propia suposición es: no mucho—. Pero la comprensión está lejos de contribuir mucho a mejorar la traducción automática.

Pregunta final: Si bien esta entrevista fue una discusión sobre su trabajo cientifico, también me gustaría incluir una pregunta de tipo más personal, si eso es apropiado. Obviamente, su ritmo de trabajo no ha disminuido mucho a pesar de haberse retirado oficialmente del MIT hace ya algunos años. Y se rumora que pronto se mudará a la Universidad de Arizona Entonces, después de todos estos años en ciencias y una carrera académica sin precedentes hasta ahora, por favor diganos, ¿qué lo mantiene activo?

Sí, me mudé a la Universidad de Arizona. Lo que me mantiene activo es la emoción del aprendizaje y el descubrimiento. Creo que los campos que siempre me han interesado abren nuevas perspectivas que ofrecen muchas esperanzas de una comprensión más profunda de la naturaleza del lenguaje y la mente.

Profesor Chomsky, muchas gracias por responder a mis preguntas. 


\section{Referencias bibliográficas}

1. Arbib, Michael A. (2017) [este número]. Language and Brain: Developmental Aspects: Eric Lenneberg at the Neurosciences Research Program in 1972. Sección Biolinguistics.

2. Becker, Misha K. (2017) [este número]. Innate Mechanisms for Acquiring Syntactic Displacement. Sección Biolinguistics.

3. Bem, Daryl J. \& Sandra L. B. (1968). Nativism Revisited: A Review of Eric H. Lenneberg's Biological Foundations of Language. Journal of the Experimental Analysis of Behavior, 11(4), 497-501. doi:10.1901/ jeab.1968.11-497.

4. Berwick, R. C. \& Chomsky, N. (2016). Why Only Us? Language and Evolution. Cambridge, MA: MIT Press.

5. Berwick, R. C. \& Stabler, E. (In press). Parsing with Minimalist Grammars. Oxford, England: Oxford University Press. [Berwick, Robert Edward Stabler (Ed.) 2019. Minimalist Parsing. NewYork, Oxford University Press.]

6. Bizzi, E. \& Ajemian, R. (2015). A Hard Scientific Quest: Understanding Voluntary Movements. Daedalus, 144(1), 83-95. Doi: 10.1162/DAED a 00324.

7. Boeckx, C. \&. Grohmann, K. (2007). The Biolinguistics Manifesto. Biolinguistics, 1, 1-8.

8. Chesi, C. \& Moro, A. (2015). The Subtle Dependency between Competence and Performance. In Angel J. Gallego \& Dennis Ott (Eds.), 50 Years Later: Reflections on Chomsky’s Aspects. Cambridge, MA: MITWPL. 33-45.

9. Chomsky, N. (1957/2002). Syntactic Structures (2nd edition.). Berlin: De Gruyter.

10. Chomsky, N. (1959). A Review of B. F. Skinner's Verbal Behavior. Language, 35(1), 26-58.

11. Chomsky, N. (2016). What Kind of Creatures Are We? New York: Columbia University Press.

12. Christiansen, M. H., \& Muller, R-A. (2015). Cultural Recycling of Neural Substrates during Language Evolution and Development. In M. S. Gazzaniga \& G. R. Mangun (Eds.), The Cognitive Neurosciences V. (pp. 675-682). Cambridge, MA: MIT Press.

13. Friederici, A. D. (2017a). Language in Our Brain: The Origins of a Uniquely Human Capacity. Cambridge, MA: MIT Press.

14. Friederici, A. D. (2017b) [este número]. Neurobiology of Syntax as the Core of Human Language 325-338. Sección Brief.

15. Friederici, A. D., Chomsky, N., Berwick, R. C., Moro, A. \& Bolhuis, J. J. (2017). Language, Mind and Brain. Nature Human Behaviour. Doi: 10.1038/s41562-017-0184-4.

16. Hauser, M. D. \& Watumull, J. (2016). The Universal Generative Faculty: The Source of Our Expressive Power in Language, Mathematics, Morality, and Music. Journal of Neurolinguistics. DOI:10.1016/j.jneuroling.2016.10.005. 17. Lashley, K. S. (1951). The Problem of Serial Order in Behavior. In L. A. Jeffress (Ed.), Cerebral Mechanisms in Behavior (pp. 112-146). New York: Wiley. 
Sobre el estudio biológico del lenguaje 50 años después. Una conversación con Noam Chomsky

18. Lenneberg, E. H. (1964). A Biological Perspective of Language. In E. H. Lenneberg (Ed.), New Directions in the Study of Language (pp. 65-88). Cambridge, MA: MIT Press.

19. Lenneberg, E. H. (1967). Biological Foundations of Language. New York: Wiley.

20. Lenneberg, E. H. (1972). Language and Brain: Developmental Aspects. A Report Based on an NRP Work Session Held November 19-21, 1972, and updated by participants. Neuroscience Research Programme Bulletin, 12(4), 511-656.

21. Marr, D. (1982/2010). Vision: A Computational Investigation into the Human Representation and Process. Cambridge, MA: MIT Press.

22. Martins, P. T. \& Boeckx, C. (2016). What We Talk about when We Talk about Biolinguistics. Linguistics Vanguard, 2(1). doi: 10.1515/lingvan-2016-0007.

23. Piattelli-Palmarini, M. (2017) [este número]. From Zero to Fifty: Considerations on Eric Lenneberg's Biological Foundations of Language and Updates, 423-444, sección Forum

\section{Fuente adicional}

24. Merriam-Webster Dictionary (2021). Expatation. https://www.merriam-webster.com/dictionary/exaptation 


\section{Información sobre los traductores}

Miguel Ángel Mahecha Bermúdez (Universidad Surcolombiana, Colombia): Docente del área de Lingüística, del programa de Literatura y Lengua Castellana, de la Facultad de Educación de la Universidad Surcolombiana (Colombia).Correo electrónico: miguelangel.mahecha@usco.edu.co

Rubén Arboleda Toro (Universidad Nacional de Colombia): Estudios de Maestría y Doctorado en Lingüística General y del Español en el Instituto Caro y Cuervo y la Universidad Nacional Autónoma de México, respectivamente. Profesor pensionado de la Universidad Nacional de Colombia. Actualmente, tiene una investigación independiente en curso acerca de las teorías sintácticas y los modelos de sintaxis generativa, de la cual se derivará un libro. Correo electrónico: rarboltoro@yahoo.com 